\title{
Chaotic Behaviors of a Digital Filter with Two's Complement Arithmetic and Arbitrary Initial Conditions and Order
}

\author{
BINGO WING-KUEN LING, WAI-FUNG HUNG and PETER KWONG-SHUM TAM \\ Department of Electronic and Information Engineering \\ The Hong Kong Polytechnic University \\ Hung Hom, Kowloon, Hong Kong, China
}

\begin{abstract}
This letter shows some counter-intuitive simulation results that the symbolic sequences and the state variables of a digital filter with two's complement arithmetic and arbitrary initial conditions and order will be eventually zero when all the filter parameters are even numbers, no matter the system matrix of the filter is stable or not.
\end{abstract}

\section{Introduction}

It is well known that chaotic behavior may occur in both a second-order digital filter [Chua, 1988, 1990b; Galias, 1992; Kocarev, 1993, 1996; Wu, 1993; Yu, 2001] and a third-order digital filter [Chua, 1990a] with two's complement arithmetic. Similar results are found in a second-order digital filter with other nonlinearities, such as a saturation-type nonlinearity [Galias, 1990] and a quantization-type nonlinearity [Lin, 1991].

However, it is found in this letter that chaotic behavior does not occur when all the filter parameters are even numbers, no matter what the initial conditions, the order and the stability of digital filter are. The main results are shown in section 2 , and a conclusion is summarized in section 3 .

\section{Simulation Results}

Consider the following $N^{\text {th }}$ order digital filter with two's complement arithmetic:

$x_{j}(k+1)=x_{j+1}(k)$ for $j=1,2, \cdots, N-1$ and $k \geq 0$

$x_{N}(k+1)=f\left(\sum_{j=1}^{N} a_{j} \cdot x_{j}(k)\right)=\sum_{j=1}^{N} a_{j} \cdot x_{j}(k)+2 \cdot s(k)$ for $k \geq 0$ 
where

$f(v)=v-2 \cdot n$ such that $-1 \leq f(v)<1$ and $n \in \boldsymbol{Z}$

$a_{j} \in \mathfrak{R}$ for $j=1,2, \cdots, N$

$-1 \leq x_{j}(k)<1$ for $j=1,2, \cdots, N$ and $k \geq 0$

and $s(k) \in\{-m, \cdots,-1,0,1, \cdots, m\}$ for $k \geq 0$

in which $m$ is the minimum integer satisfying

$-2 \cdot m-1 \leq \sum_{j=1}^{N} a_{j} \cdot x_{j}(k)<2 \cdot m+1$

According to our intensive simulations, we have the following observation:

\section{Observation.}

If $a_{j}$ is an even number and $\left|x_{j}(0)\right|<1$ for $j=1,2, \cdots, N$, then $\exists k_{0} \in \boldsymbol{Z}^{+} \cup\{0\}$ such that $x_{j}(k)=0$ and $s(k)=0$ for $\forall k \geq k_{0}$ and $j=1,2, \cdots, N$.

If $\exists j \in\{1,2, \cdots, N\}$ such that $a_{j}$ is not an even number, and the system matrix of the digital filter is unstable, then chaotic behavior may occur.

To understand this phenomenon, we model the filtering process as a sum of Bernoulli shift operations. Since, for practical implementation, any number is represented by only a finite number of bits, the initial condition can be represented in a binary form as follows:

$\left|x_{j}(0)\right|=\sum_{n=1}^{P} p_{n, j} \cdot 2^{-n}$

where $p_{n, j} \in\{0,1\}$ for $j=1,2, \cdots, N$

and $P$ is the number of bits, not including the sign bit, for representing the state variables.

Since $a_{j}$ are even numbers, we can let:

$\left|a_{j}\right|=\sum_{n=1}^{M} \alpha_{n, j} \cdot 2^{n}$

where $\alpha_{n, j} \in\{0,1\}$ for $j=1,2, \cdots, N$

and $M$ is the number of bits, not including the sign bit, for representing the filter coefficients. We have 
$\sum_{j=1}^{N} a_{j} \cdot x_{j}(k)=\sum_{j=1}^{N}\left(\left(\sum_{n=1}^{M} \alpha_{n, j} \cdot 2^{n}\right) \cdot\left(\sum_{n=1}^{P} p_{n, j} \cdot 2^{-n}\right)\right)$

$=\sum_{j=1}^{N}\left(s_{j}^{\prime}+\sum_{i=1}^{P-1} \beta_{i, j} \cdot 2^{-i}\right)$

where $s_{j}^{\prime} \in \boldsymbol{Z}^{+} \bigcup\{0\}$

Since the summation in $\sum_{i=1}^{P-1} \beta_{i, j} \cdot 2^{-i}$ is from $i=1$ to $i=P-1$, the most significant bit is absorbed in $s_{j}^{\prime}$ after the first iteration, and all the bits will vanish after $P$ iterations. Therefore, the state trajectories will eventually converge to some origin.

To demonstrate the observation, a third-order and a fourth-order digital filter with two's complement arithmetic is shown. Results of higher order digital filter with two's complement arithmetic can be obtained similarly.

Figure 1 shows the state variables and symbolic sequence of a third-order digital filter with two's complement arithmetic when the filter parameters and the initial conditions are randomly generated from a set of even numbers and the set $[-1,1)$, respectively. It can be seen from the figure that when the filter parameters are even numbers, though the system matrix is unstable, the values of the symbolic sequence and the state variables will be eventually zero. Similarly, figure 2 shows the cases with the same initial condition as that in figure 1, but the filter parameters are deviated slightly from those in figure 1 . In this case, chaotic behavior occurs. Similar results for the fourth-order cases are shown in figure 3 and figure 4, respectively.

\section{Conclusion}

In this letter, we report some counter-intuitive simulation results that the symbolic sequences and the state variables of a digital filter with two's complement arithmetic and arbitrary initial conditions and order will be eventually zero when all the filter parameters are even numbers, no matter the system matrix of the filter is stable or not.

\section{Acknowledgement}

The work described in this letter was substantially supported by The Hong Kong Polytechnic University. 


\section{References}

[1] Chua, L. O. \& Lin, T. [1988], "Chaos in digital filters," IEEE Transactions on Circuits and Systems, vol. 35, no. 6, pp. 648-658.

[2] Chua, L. O. \& Lin, T. [1990a], "Chaos and fractals from third-order digital filters," International Journal of Circuit Theory and Applications, vol. 18, pp. 241-255.

[3] Chua, L. O. \& Lin, T. [1990b], "Fractal pattern of second-order non-linear digital filters: a new symbolic analysis," International Journal of Circuit Theory and Applications, vol. 18, pp. 541-550.

[4] Galias, Z. \& Ogorzalek, M. J. [1990], "Bifurcation phenomena in second-order digital filter with saturation-type adder overflow characteristics," IEEE Transactions on Circuits and Systems, vol. 37, no. 8, pp. 1068-1070.

[5] Galias, Z. \& Ogorzalek, M. J. [1992], "On symbolic dynamics of a chaotic second-order digital filter," International Journal of Circuit Theory and Applications, vol. 20, pp. 401-409.

[6] Kocarev, L. \& Chua, L. O. [1993], "On chaos in digital filters: case $b=-1$," IEEE Transactions on Circuits and Systems-II: Analog and Digital Signal Processing, vol. 40, no. 6, pp. 404-407.

[7] Kocarev, L., Wu, C. W. \& Chua, L. O. [1996], "Complex behavior in digital filters with overflow nonlinearity: analytical results," IEEE Transactions on Circuits and Systems-II: Analog and Digital Signal Processing, vol. 43, no. 3, pp. 234-246.

[8] Lin, T \& Chua, L. O. [1991], "On chaos of digital filters in the real world," IEEE Transactions on Circuits and Systems, vol. 38, no. 5, pp. 557-558.

[9] Wu, C. W. \& Chua, L. O. [1993], "Properties of admissible symbolic sequences in a second-order digital filter with overflow non-linearity," International Journal of Circuit Theory and Applications, vol. 21, pp. 299-307.

[10] Yu, X. \& Galias, Z. [2001], "Periodic behaviors in a digital filter with two's complement arithmetic," IEEE Transactions on Circuits and Systems-II: Analog and Digital Signal Processing, vol. 48, no. 10, pp. 1177-1190. 

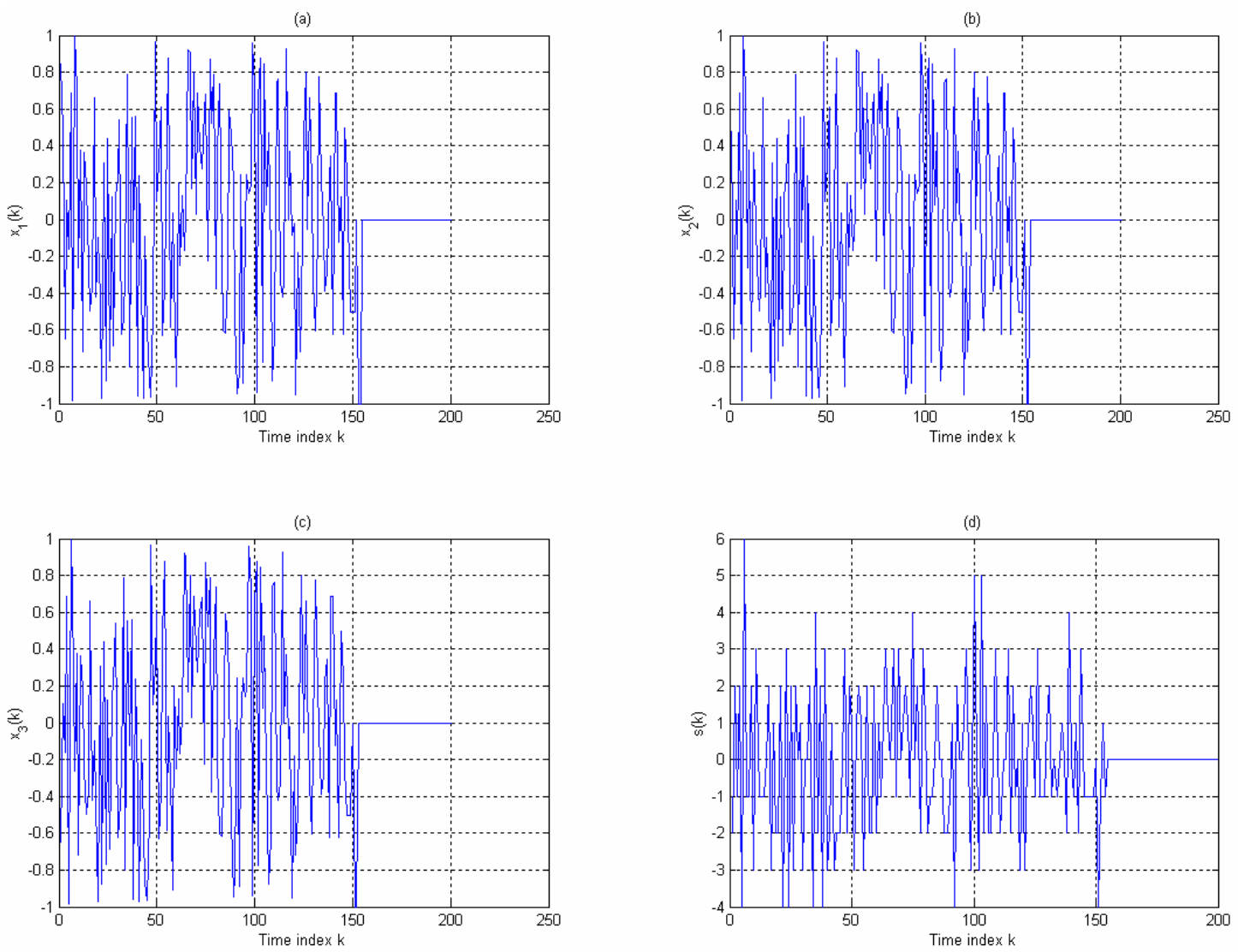

Fig. 1. State variables and symbolic sequences of a third-order digital filter with two's complement arithmetic, $\mathbf{x}(0)=\left[\begin{array}{lll}0.8436 & 0.4764 & -0.6475\end{array}\right]^{T}, a_{1}=-2, a_{2}=4$ and $a_{3}=-6$. (a) State variable $x_{1}(k)$. (b) State variable $x_{2}(k)$. (c) State variable $x_{3}(k)$. (d) Symbolic sequence $s(k)$. 

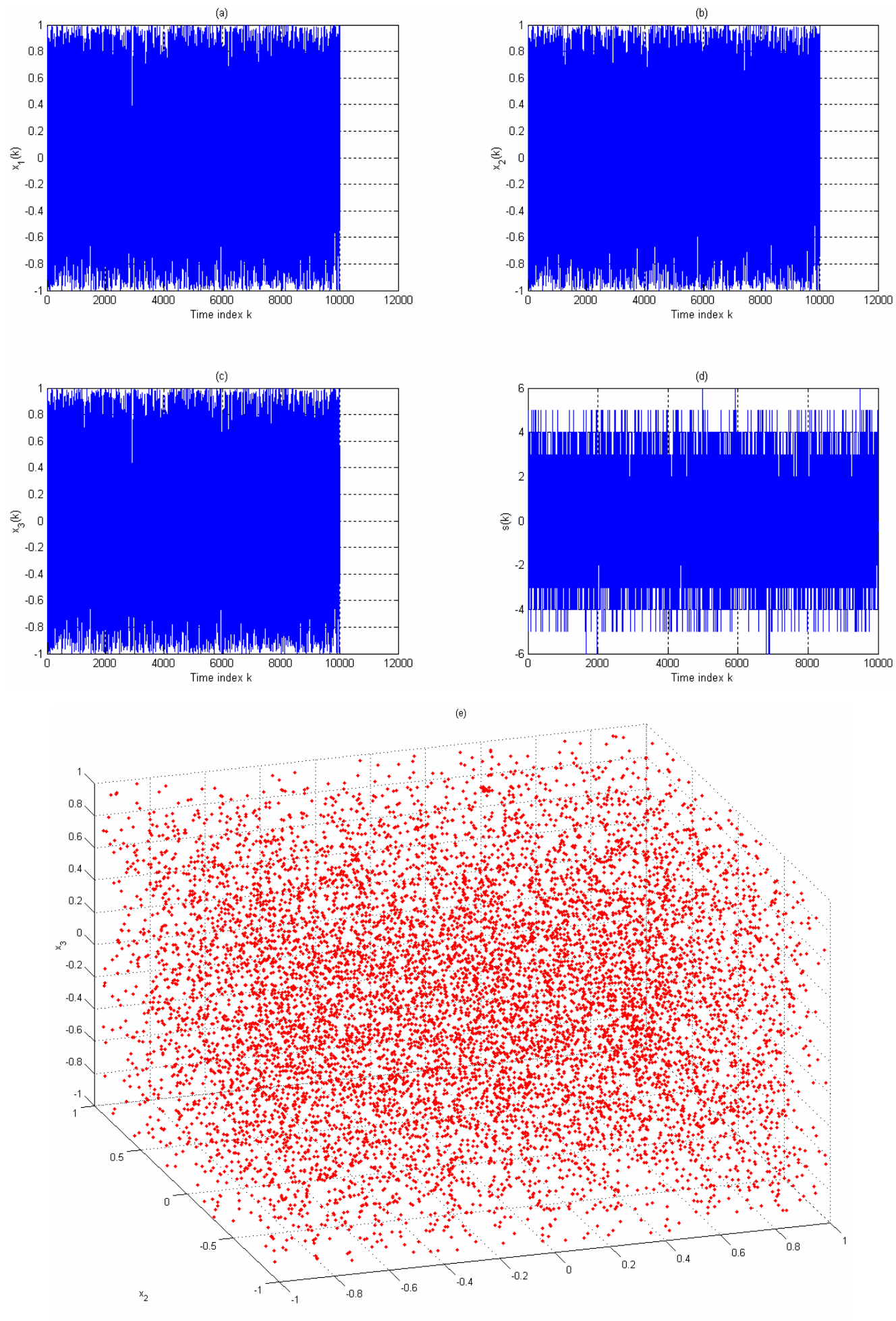

Fig. 2. State variables, symbolic sequences and phase portrait of a third-order digital filter with two's complement arithmetic, $\mathbf{x}(0)=\left[\begin{array}{llll}0.8436 & 0.4764 & -0.6475\end{array}\right]^{T}$, $a_{1}=-1.99, a_{2}=4.01$ and $a_{3}=-5.99$. (a) State variable $x_{1}(k)$. (b) State variable $x_{2}(k)$. (c) State variable $x_{3}(k)$. (d) Symbolic sequence $s(k)$. (e) Phase portrait. 

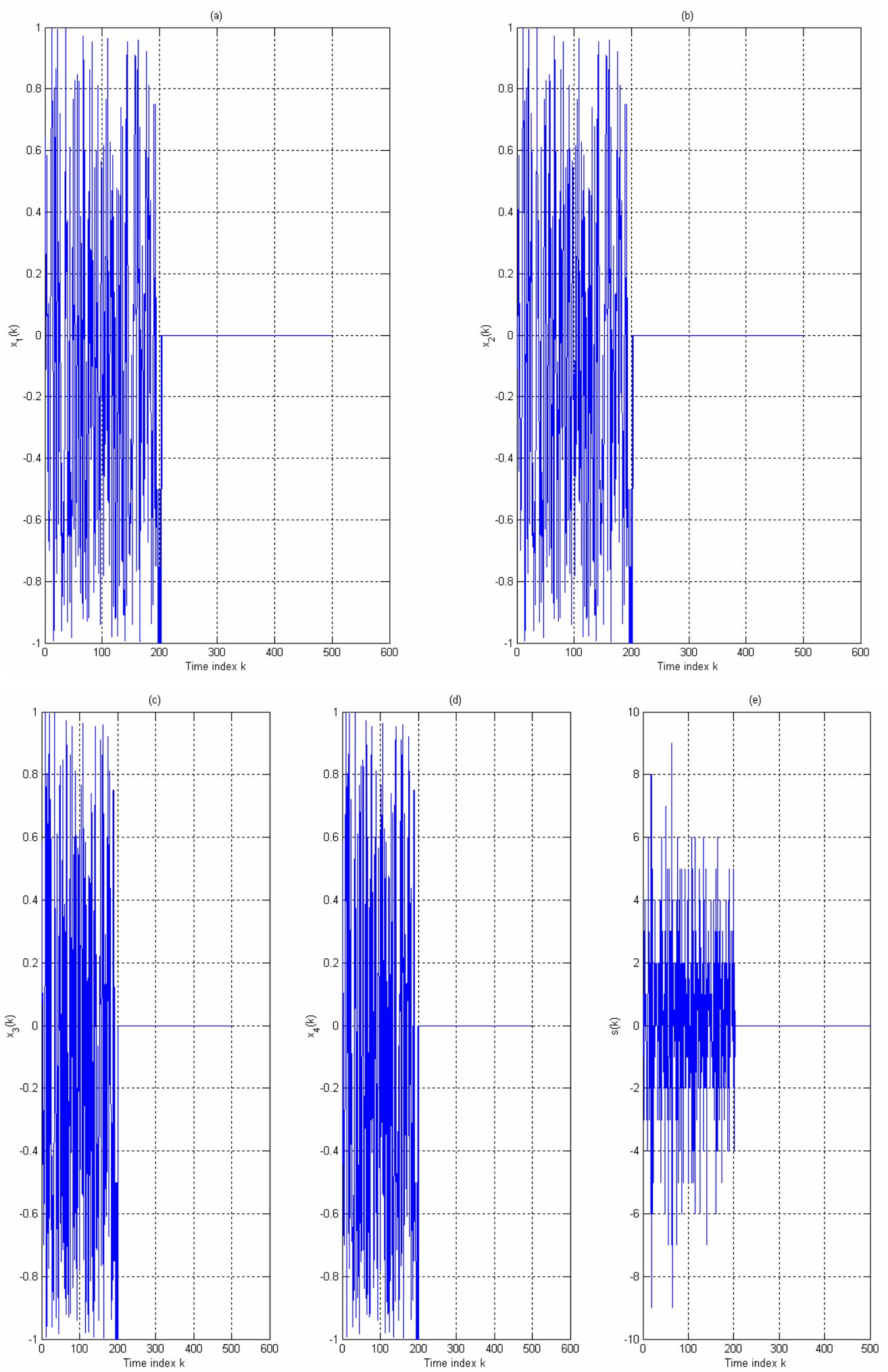

Fig. 3. State variables and symbolic sequences of a fourth-order digital filter with two's complement arithmetic, $\mathbf{x}(0)=\left[\begin{array}{llll}0.6428 & -0.1106 & 0.2309 & 0.5839\end{array}\right]^{T}, a_{1}=-2, a_{2}=4$, $a_{3}=-6$ and $a_{4}=8$. (a) State variable $x_{1}(k)$. (b) State variable $x_{2}(k)$. (c) State variable $x_{3}(k)$. (d) State variable $x_{4}(k)$. (e) Symbolic sequence $s(k)$. 

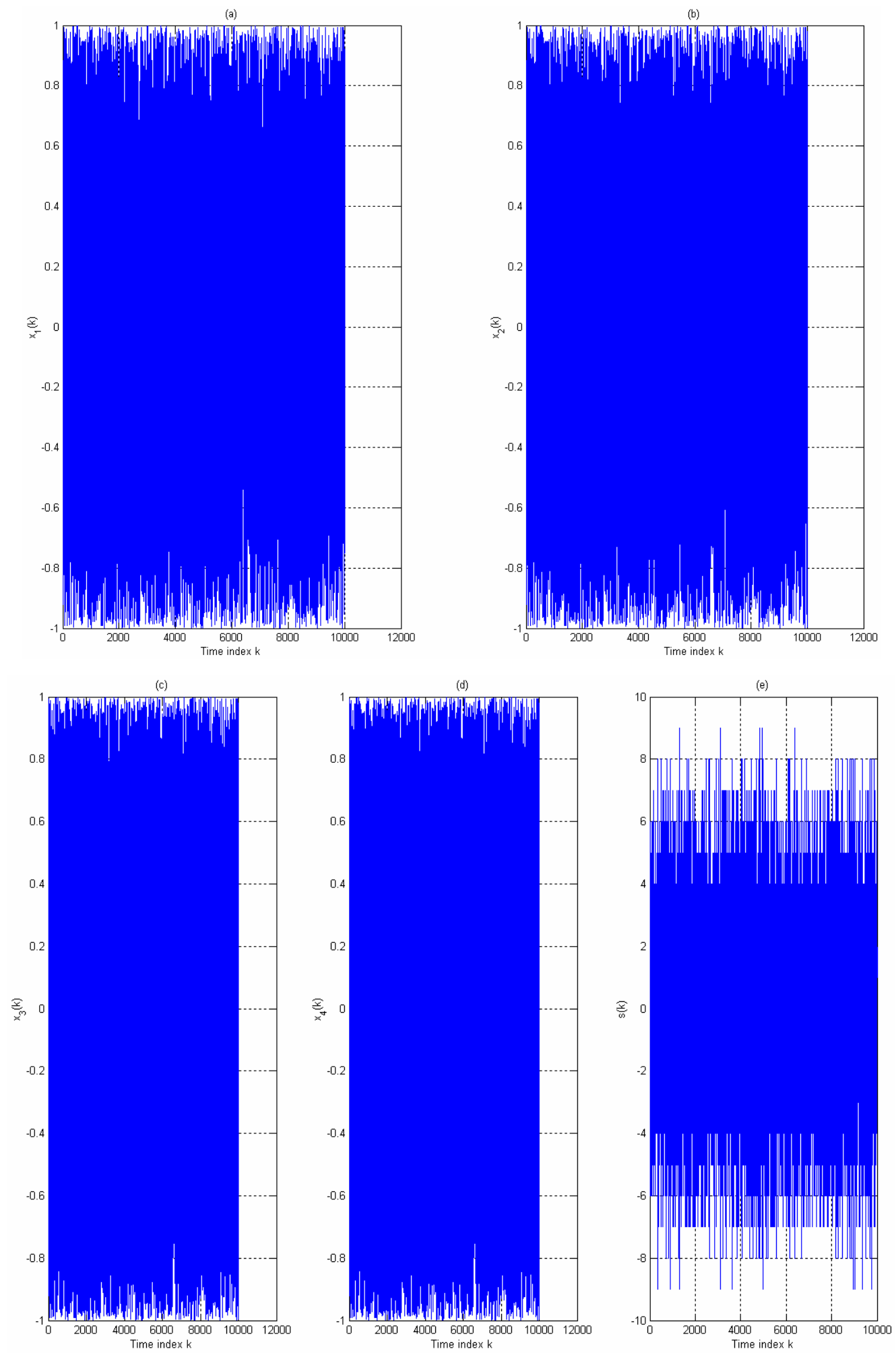

Fig. 4. State variables and symbolic sequences of a fourth-order digital filter with two's complement arithmetic, $\mathbf{x}(0)=\left[\begin{array}{llll}0.6428 & -0.1106 & 0.2309 & 0.5839\end{array}\right]^{T}, \quad a_{1}=-2.01$, $a_{2}=4.01, a_{3}=-6.01$ and $a_{4}=8.01$. (a) State variable $x_{1}(k)$. (b) State variable $x_{2}(k)$. (c) State variable $x_{3}(k)$. (d) State variable $x_{4}(k)$. (e) Symbolic sequence $s(k)$. 